\title{
The Application of an Artificial Neural Network to Support Decision Making in Edentulous Maxillary Implant Prostheses
}

\author{
Leyla Sadighpour ${ }^{1}$, Susan Mir Mohammad Rezaei ${ }^{1}$, Mojgan Paknejad ${ }^{2}$, \\ Fatemeh Jafary ${ }^{1}$ and Pooya Aslani ${ }^{1}$ \\ ${ }^{1}$ Department of Prosthodontics, Faculty of Dentistry, Tehran University of Medical Sciences, Tehran, Iran \\ ${ }^{2}$ Department of Periodontics, Faculty of Dentistry, Tehran University of Medical Sciences, Tehran, Iran
}

Correspondence should be addressed to: Leyla Sadighpour; sedighle@tums.ac.ir

Received 29 June 2013; Accepted 31 July 2013; Published 24 January 2014

Academic Editor: Ricardo Faria Ribeiro

Copyright (C 2014 Leyla Sadighpour, Susan Mir Mohammad Rezaei, Mojgan Paknejad, Fatemeh Jafary and Pooya Aslani. Distributed under Creative Commons CC-BY 3.0

\begin{abstract}
This article presents the development of an artificial neural network to support the clinical decision making on the maxillary implant restoration for the patient with edentulous maxilla.

The records of 47 patients were included in the study. The clinical data were presented to two faculty prosthodontists to point out most relevant parameters in determining the final treatment option. The collected data were codes as input criteria. The data of 35 patients were introduced to a designed neural network with feed forward /back propagation architecture. The testing phase was followed by presenting 12 new cases to the network and the accuracy was measured. The network performed well within learning rate of 0.005.The accuracy of network for the new cases was 83.3 $\%$. An artificial neural network trained with clinical scenarios could perform well as a decision making support system and may offer the potentials in clinical education.
\end{abstract}

Keywords: Artificial neural network, decision making, maxillary implant.

\section{Introduction}

The development of a sound and defensible treatment plan is an important core competency for every dental graduate. Banning (2008) indicated that dental practitioners must incorporate their knowledge, evidence, and experience in formulating appropriate treatment plans for their patients. According to Crespo et al. (2004) however, in practice the performance of novice and expert dentists differ distinctly in terms of their clinical reasoning skills, ability to diagnose patients, and adeptness at treatment plan development. Wainwright et al. (2011) found that continuous exposure to

Cite this Article as: Leyla Sadighpour, Susan Mir Mohammad Rezaei, Mojgan Paknejad, Fatemeh Jafary and Pooya Aslani (2014), "The Application of an Artificial Neural Network to Support Decision Making in Edentulous Maxillary Implant Prostheses," Journal of Research and Practice in Dentistry, Vol. 2014 (2014), Article ID 369025, DOI: 10.5171/2014.369025 
similar clinical scenarios enables experts to recognize the pattern or script of an illness, and that this could be used as a pattern for a decision-support system. Lisboa (2002) and Mendonca (2004) reviewed computerized expert systems such as artificial neural networks (ANNs) that have been used as decision making support systems in several medical specialties. As these systems are trained by using real clinical scenarios and expertise, they appear as if the experts' minds have been modeled. These could provide other practitioners or students with the information and support they need to establish their clinical performance on best practice-based evidence.

In a brief introduction regarding the use of neural networks in medical science, Brickley and Shepherd (1998), Dey and Dey (2002) and Amato et al (2013) discussed the hypothesis that artificial neural networks attempt to simulate the human brain functions of learning and experience. It is believed that each learning process (or experience) can affect the interconnection (synapses) of the neurons in the brain and nervous system. A memory is therefore built up by strengthening or weakening the synaptic connections to specific neuron(s). Accordingly, each ANN consists of several nodes (equivalent to neurons) that are arrayed in layers. The type of ANN architecture that is typically applied to medicine and dentistry is the multi-layer perceptron, also known as a multi-layer neural network (MLN), with a feedforward/back-propagation topology. The results of studies by Ghiassi and Saidane (2005) and Panchal et al. (2011) showed that an MLN is capable of defining the function that relates the input data to the desired response. Hence, MLNs are useful in pattern recognition, classification, and prediction. In each learning cycle, a set of data is introduced to the input perceptrons (neurons or nodes). The connection between the perceptrons allows a one-way flow of data from one unit to other units of the hidden layer, through which a signal is generated which is transferred to the output layer. If the output response does not match the known output within a reasonable time limit, then an error signal is propagated back through the network. This signal adjusts the weight of the network connections until the desired response is achieved. An MLN with a feed-forward /back-propagation algorithm for learning is easy to use and can potentially map any complex nonlinear input/output relationship.

The application of ANNs to the field of dentistry has been reviewed by Vikram and Karjodkar (2009). In general, ANNs are helpful in predicting clinical situations in which a strong rule-based relationship is not sufficient for a conclusive decision to be made and the final decisions are, in part, subjective. Brickley and Shepherd (1996) compared the performance of an ANN and a selected group of oral surgeons in making decisions regarding third-molar removal. They found a high degree of agreement between the senior surgeons and the trained network. In addition, Devito et al. (2008) developed an ANN for the diagnosis of proximal dental caries and found that the use of an MLN could improve diagnosis by $39 \%$. Oladokun et al. (2008) developed an ANN to assess student academic performance, although this was related to non-medical students. The aim of the present study was to develop an ANN trained by a series of restorative cases. The treatment of edentulous maxilla by implant prostheses was selected as the model, because the choice of the design of the final prosthesis is not straightforward and depends on several clinical parameters of each patient.

\section{Methods}

The records of patients who attended the implant dentistry division of Tehran University of Medical Sciences were examined, and 47 clinical records of edentulous maxilla were selected. Records with incomplete data regarding patient examination or surgical procedure 
information were excluded. Maxillofacial patients were also excluded. Several authorities, Misch (2005), Zitzman and Marinella (2000), and Jivraj et al (2006) discussed an average of 15 factors that should be considered regarding the treatment planning of an edentulous maxilla with dental implants. The priorities of each factor (weighting) in the treatment-planning sequences were not initially determined accurately. Since, as the number of input factors increases, the number of cases required for teaching the ANN also increases, the collected data were presented to two expert prosthodontists, both of whom were faculty members with more than 10 years of experience in implant dentistry. They were asked to address the specific factors that affect their decision on the final prosthetic design for each patient with consideration of the standards presented by Misch (2005), Zitzman (2000), and Jivraj et al (2006). The number of factors was then limited to the most relevant ten; and factors such as implant length and demographic data were omitted. All of the factors related to aesthetics were also summarized as favorable or unfavorable. The approved input criteria were coded as shown in Table 1.

Table 1. Clinical Information Used for Input and their Codes. Items 1 to 7 were Used to Indicate the Distribution of Inserted Implants

\begin{tabular}{|c|c|c|c|c|}
\hline Input & Name & Description & Coding method & Range \\
\hline 1 & IMP_ANT & $\begin{array}{l}\text { The number of implant in } \\
\text { incisors region }\end{array}$ & Numerical & $0-4$ \\
\hline 2 & IMP_RC & $\begin{array}{l}\text { The number of implant in } \\
\text { the right canine region }\end{array}$ & Numerical & $0-1$ \\
\hline 3 & IMP_LC & $\begin{array}{l}\text { The number of implant in } \\
\text { the left canine region }\end{array}$ & Numerical & $0-1$ \\
\hline 4 & IMP_RP & $\begin{array}{l}\text { The number of implant in } \\
\text { the right premolars region }\end{array}$ & Numerical & $0-2$ \\
\hline 5 & IMP_LP & $\begin{array}{l}\text { The number of implants in } \\
\text { the left premolars region }\end{array}$ & Numerical & $0-2$ \\
\hline 6 & IMP_RM & $\begin{array}{l}\text { The number of implant in } \\
\text { the right molars region }\end{array}$ & Numerical & $0-2$ \\
\hline 7 & IMP_LM & $\begin{array}{c}\text { The number of implants in } \\
\text { the left molars region }\end{array}$ & Numerical & $0-2$ \\
\hline 8 & Esthetics & Esthetics related factors & $\begin{array}{c}\text { Logical } \\
\text { Favorable }=1 \\
\text { Unfavorable }=0\end{array}$ & 0,1 \\
\hline 9 & $\mathrm{OAO}$ & $\begin{array}{c}\text { The type of dentition in the } \\
\text { opposing arch }\end{array}$ & $\begin{array}{c}\text { Ordinal scale } \\
\text { Natural dentition=1 } \\
\text { Implant retained fixed } \\
\text { prosthesis }=1 \\
\text { Complete denture }=2 \\
\text { Partial removable } \\
\text { denture }=3 \\
\text { Overdenture (implant or } \\
\text { teeth supported) }=3\end{array}$ & $0-3$ \\
\hline 10 & Inter Occ & The interoclusal distance & Millimeters & $8-20$ \\
\hline
\end{tabular}


The network architecture was then designed. An MLP topology with one input layer, one hidden layer, and one output layer was chosen. According to Panchal et al (2011), the number of hidden layer nodes could be up to $2 / 3$ of the sum of the number of input layer nodes and the output layer nodes. Yet it must be less than twice the size of the input layer. The number of neurons in the hidden layer has a profound impact on the system performance and is not easily determined. An inadequate number of neurons will lead to failure in modeling complex data, a phenomenon known as underfitting. However, including too many neurons results in overfitting, in which the input data sets are not sufficiently large to pass through all the available nodes and weight them accordingly. Under these circumstances, the system will not converge over a reasonable time. If it does, its accuracy for the trained case would be high, but with a poor capacity for dealing with new cases. Hence, selecting the number of nodes in the hidden layer is an iterative trial-and-error process. Following the studies of Ghiassi and Saidane (2005) and Panchal et al (2011), a hidden layer with 20 units was selected for the initial stage (Figure 1) and a computer program was written in the FORTRAN programming language.

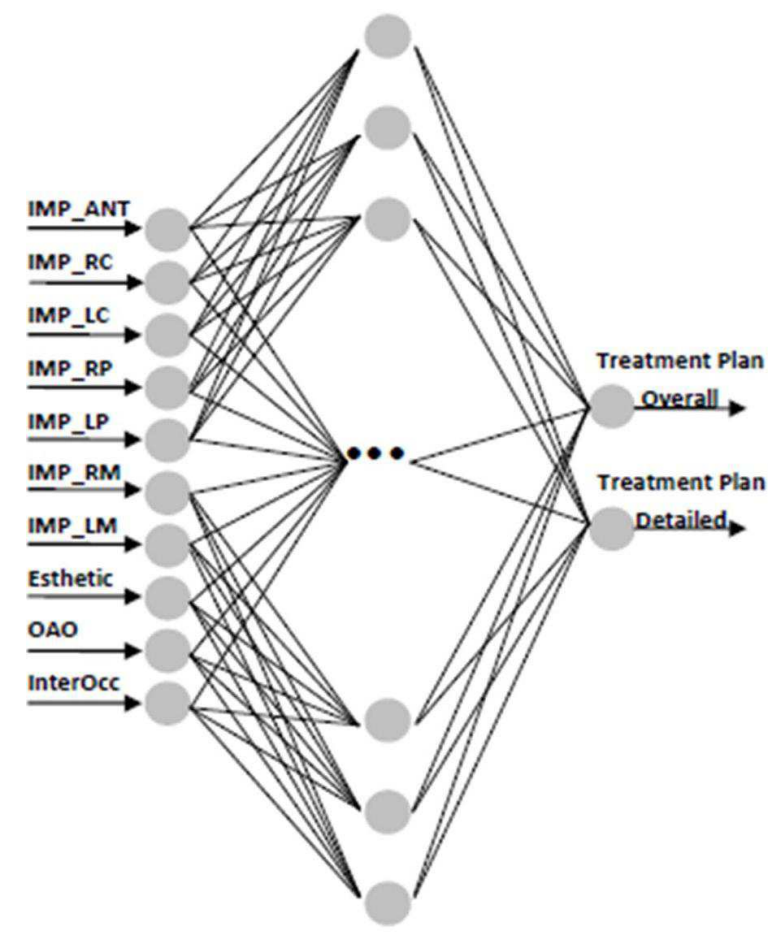

Fig 1. The Architecture of the Designed ANN. Each Input Node Connects to a Node in Hidden Layer and Each Node in Hidden Layer Connects to a Node in Output Layer

The learning process in the ANN followed a feed-forward/back-propagation algorithm. Therefore, it was necessary that the final prosthesis design be introduced to the network as the desired output (Table 2) .Two examples of the treatment choices are shown in Figures 2 and 3. 
Table 2. The Final Treatment Options for Output Response

\begin{tabular}{|c|c|c|c|}
\hline Output & Name & Coding method & Range \\
\hline 1 & $\begin{array}{c}\text { Overall } \\
\text { treatment }\end{array}$ & Ordinal & $\begin{array}{c}\text { Removable }=1 \\
\text { Fixed }=2\end{array}$ \\
\hline 2 & Detailed treatment & Ordinal & $\begin{array}{c}\text { Removable }- \text { bar attachment }=1 \\
\text { Removable }- \text { bal attachment }=2 \\
\text { Removable }- \text { bar or ball }=3 \\
\end{array}$ \\
& & & $\begin{array}{c}\text { Fixed }- \text { hybrid }=4 \\
\text { Fixed }- \text { PFM }=5\end{array}$ \\
\hline
\end{tabular}

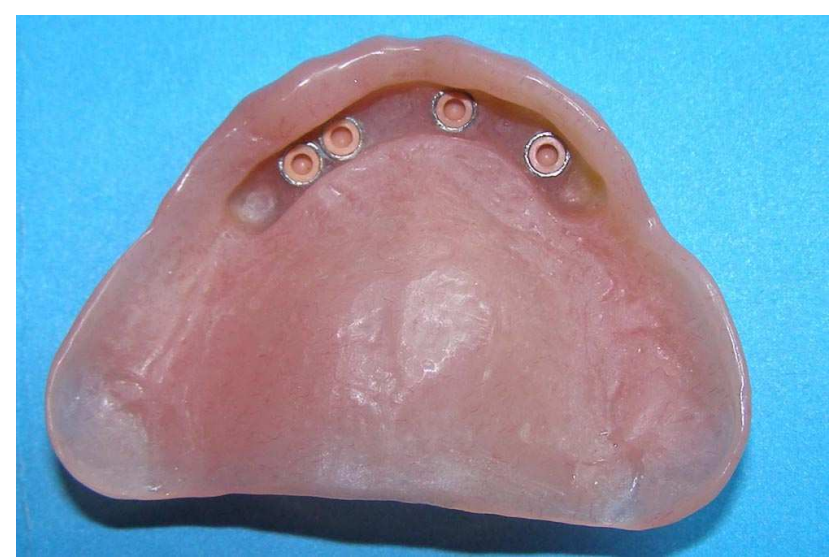

Fig 2, a. An Edentulous Maxilla with 4 Implants Inserted in Left Canine, Left Lateral Insicor, Right Central Incisor and Right First Premolar Location

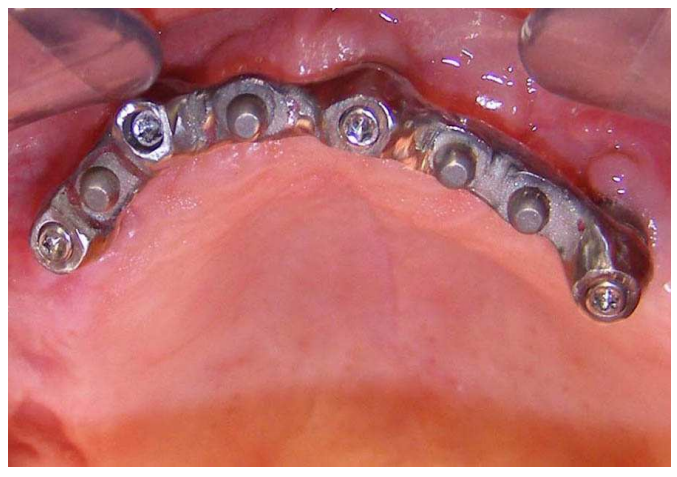

Fig 2, b. A Bar-Ball Superstructure was Fabricated 


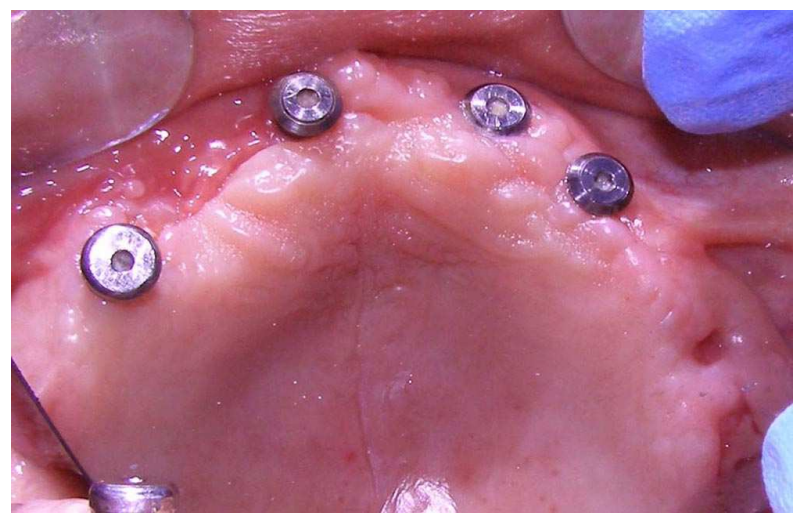

Fig2, c. The Removable Bar-Ball Denture was Made

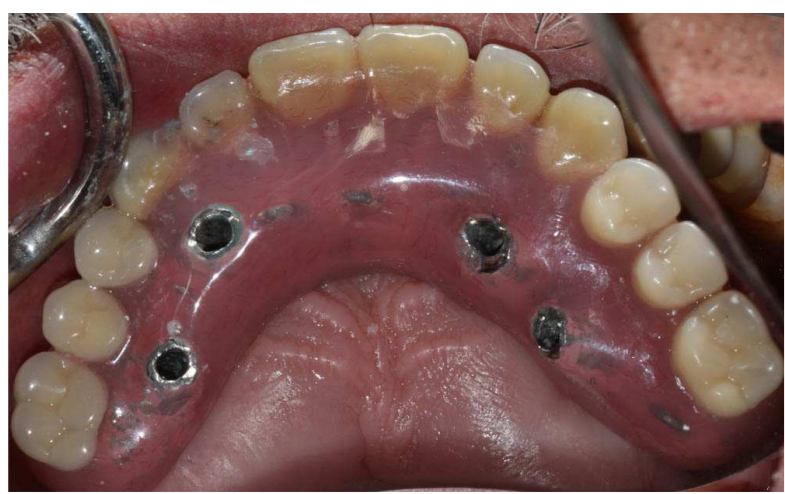

Fig 3. An Example of Fixed Hybrid Prosthesis

For each teaching cycle, the error (difference between the generated output and actual result) was propagated backward through the network to adjust the weightings of the nodes and connections in the system. The accuracy within the system would then be recalculated over the training data, until the minimum acceptable error was reached i.e. the system output matched the real treatment option that was made for the specific patient. The pace of performing such a task cycle is called the training rate. If the system architecture is designed optimally, the output converges toward a defined outcome; otherwise it would oscillate, which is considered as failure.

Generally, there is no rule-of-thumb for finding the appropriate training rate. Training rate selection is a tradeoff between the processing speed and the accuracy of the output response. Based on information from previous studies, learning rates of 0.0005 , 0.005 , and 0.05 were tested to optimize the convergence of the data processing in the network while minimizing error. Of the 47 of patients' records, thirty-five cases were used to train the ANN, and about $20 \%$ of the teaching cases (12 cases) were set aside to test the network performance. The accuracy of the network performance was calculated as the percentage of the correct responses by the network compared with the actual treatment that the patient had received.

\section{Results}

The ANN training phase was performed successfully. According to the data processing by the ANN, the transfer function, 
which was the weighted algorithm of the connections of the nodes, was a hyperbolic tangent. The learning rate of the network was optimized at 0.005 for 35 input cases (Figure 4). There was no need to change the number of nodes in the hidden layer. The testing phase of the ANN showed an $83.3 \%$ successful response ( 9 out of 12 cases). Two

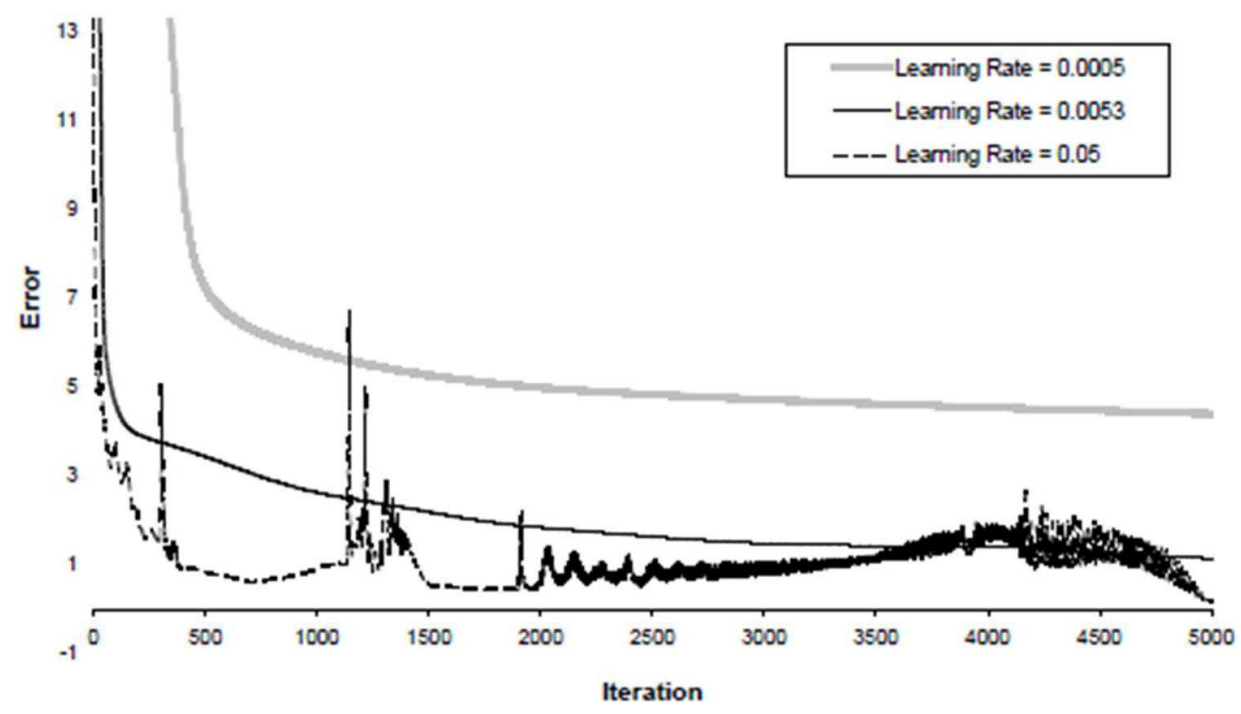

Fig 4. The Performance of the Network within Determined Rates

\section{Discussion}

An ANN was developed to predict the treatment plan for restoring an edentulous maxilla with dental implants. As explained by Mendonca (2004), such a network could be used as a decision-support system, to aid students and novice dentists in the application of best practice-based evidence to patient management. Given the high failure rate of implant-retained prostheses reported by Jemt and Johansson (2006), the use of a trained ANN with actual cases may enhance the quality of care and overall patient satisfaction. In the teaching phase, the network performed well in terms of converging to the correct response over a reasonable time. The accuracy of the network response in new cases was $83.3 \%$. The network architecture was established with 9 inputs, one hidden layer with 20 nodes; and of the three unmatched responses were related to the type of attachment used in the prostheses. In the third case, the actual treatment was a hybrid fixed prosthesis, while the calculated response of the ANN was a removable prosthesis. The unsuccessful response was related to the selection of the type of attachment in the prostheses. 
input and output factors can display many different behaviors, a large number of teaching cases may be required. In the present study, the number of teaching cases was selected empirically as a power of 2 of the input criteria. As the number of available patients who would satisfy the inclusion criteria was limited, the input-output relationship was adjusted to make the learning process less complicated. The results of our study are difficult to compare with other studies due to the specificity of the trained subjects. For example, in a previous study by Martina et al. (2006), data from 48 patients were introduced to an ANN that had been designed for the decision support of orthodontic extraction based on cephalographic measurements. Although the teaching cases were within the range of our study, the success rate (the accuracy of the network in achieving the correct answer during the training phase) ranged from $76.5 \%$ to $87.5 \%$ when a variable number of nodes were designed into the hidden layer. In addition, the size of input in their study was 3.5 times greater than in our study, which means that unless the input/output logic terms fit into a more rule-based relationship, the training cases should be $8\left(2^{3}\right)$ times more than was used in their study to achieve a more accurate result. However, the subjectivity of the treatment choice in their study was noted to be high. In another study by Brickley and Shepherd (1996), 119 patient records were applied to an ANN with 19 inputs, the purpose of which was to make relatively complex decisions regarding third molar removal.

The results of the present investigation showed $83.3 \%$ accuracy between the ANN response and the treatments that had been delivered to the patients. The accuracy of the ANN performance could be improved by increasing the number of teaching cases, which was a limitation of our study as previously mentioned. Yet, this result is within the range given by similar previous investigations. For instance, Lopes et al. (2008) found an 88\% agreement between a trained ANN and histological examination in detecting proximal caries. An ANN for decision-making concerning orthodontic extraction showed an accuracy of $75 \%$ in a study by Martina et al. (2006). Aw et al. (1996) also found a 72\% agreement between experts and an ANN in grading dental cavities prepared by students.

In the present work, the ANN was inaccurate primarily in the selection of the attachment type. The choice between a bar or ball attachment depends on several factors, such as the intra-implant distance, the risk of the metal substructure being visible from the acrylic flange of the denture, and nonuniform interocclusal distance across the jaws. This complexity most likely influenced the decision-making ability of the ANN. Additionally, because of the limited number of available cases, several input criteria were omitted. Therefore, the ANN was unable to establish any relationship between those omitted factors and the output. A distinct advantage of ANNs is their "updating" capability (i.e., their ability to learn from new cases). Nevertheless, when the number of input units, output units, or hidden layers is changed, a new network system must be developed.

A promising application of ANNs trained by real cases lies in their ability to assess students at different educational levels. Stevens and Lopo (1994) examined a trained ANN in the evaluation of student problemsolving performances. They found promising results in the ability of the network to discriminate between novice and expert performances, particularly for more complex problems. Aw et al. (1996) found that a trained ANN could grade amalgam cavity preparation at a level that was very close to that of expert instructors. In a case-based assessment, the performance of a student could be compared with that of experts in solving the proposed clinical scenarios. One potential limitation is that a separate ANN would need to be designed and trained for each clinical problem. Our future work will 
involve investigating the efficacy of such assessment systems.

\section{Conclusion}

Within the limitations of the present study, it can be concluded that an ANN, trained by real scenarios of implant-based restorative cases, could mimic expert performance with an accuracy of up to $83 \%$.

\section{Acknowledgement}

This research was an undergraduate dissertation and supported by the Vice Chancellor for Research of Tehran University of Medical Sciences.

\section{References}

Amato, F., Lopez, A., Pena-Mendez, E. M., Vanhara, P., Hampl, A. \& Havel, J. (2013). "Artificial Neural Networks in Medical Diagnosis," Journal of Applied Biomedicine, 11 (1):47-58.

Aw, T. C., Lang, P., Yaman, P. \& Dennison, J. B. (1996). "Using a Neural Network Expert System in Grading Dental Cavity Preparations," Proceedings of the AMIA Annual Fall Symposium, (1996), 876.

Banning, M. (2008). “Clinical Reasoning and its Application to Nursing: Concepts and Research Studies," Nurse Education in Practice, 8 (3):177-83.

Brickley, M. R., Shepherd, J. P. \& Armstrong, R. A. (1998). "Neural Networks: A New Technique for Development of Decision Support Systems in Dentistry," Journal of Dentistry, 26 (4):305-9.

Brickely, M. R. \& Shepherd, J. P. (1996). "Performance of a Neural Network Trained to Make Third-Molar Treatment Planning Decisions," Medical Decision Making, 16 (2):153-60
Crespo, K. E., Torres, J. E. \& Recio, M. E. (2004). "Reasoning Process Characteristics in the Diagnostic Skills of Beginner, Competent, and Expert Dentists," Journal of Dental Education, 68 (12):1235-44.

Devito, L. K., de Souza Barbosa, F. \& Flippe Filho, W. N. (2008). "An Artificial Multilayer Perception Neural Network for Diagnosis of Proximal Dental Caries," Oral Surgery, Oral Medicine, Oral Pathology, Oral Radiology and Endodontology, 106(6):879-84.

Dey, P. \& Dey, R. (2002). “Artificial Neural Network- Mechanism and Application in Pathology," Indian journal of pathology \& microbiology, 45 (3):371-4.

Frize, M. \& Frasson, C. (2000). "DecisionSupport and Intelligent Tutoring Systems in Medical Education," Clinical and Investigative Medicine, 23 (4):266-9.

Ghiassi, M. \& Saidane, H. (2005). "A Dynamic Architecture for Artificial Neural Networks," Neurocomputing, 63 (1):397-413.

Jemt, T. \& Johansson, J. (2006). "Implant Treatment in the Edentulous Maxillae: A 15Year Follow-up Study on 76 Consecutive Patients Provided with Fixed Prostheses," Clinical Implant Dentistry and Related Research, 8 (2):61-9.

Jivraj, S., Chee, W. \& Corrado, P. (2006). "Treatment Planning of the Edentulous Maxilla," British Dental Journal 2006; 201 (5):261-279.

Lisboa, P. J. G. (2002). “A Review of Evidence of Health Benefit from Artificial Neural Networks in Medical Intervention," Neural Networks, 15 (1):11-39.

Martina, R., Teti, R., Addona, D. D. \& Iodice, G. (2006). Neural Network Based System for Decision Making Support in Orthodontic Extractions, Special Session on ICME, 2nd Int. Virtual Conf. on Intelligent Production Machines and Systems - IPROMS (2006), 3-14 July: 235-240 
Mendonca, E. A. (2004). "Clinical Decision Support Systems: Perspectives in Dentistry," Journal of Dental Education, 68 (6):589-97.

Misch, C. E. (2005). Dental Implant Prosthetics, Mosby Inc., St Louise, Missouri USA.

Oladokun, V. O., Adebanjo, A. T. \& CharlesOwaba, O. E. (2008). "Predicting Students' Academic Performance Using Artificial Neural Network: A Case Study of an Engineering Course," The Pacific Journal of Science and Technology, 9 (1):27-9.

Panchal, G., Ganatra, A., Kosta, Y. P. \& Panchal, D. (2011). "Behaviour Analysis of Multilayer Perceptrons with Multiple Hidden Neurons and Hidden Layers," International Journal of Computer Theory and Engineering, 3 (2):3327.

Stevens, R. H. \& Lopo, A. C. (1994). "Artificial Neural Network Comparison of Expert and Novice Problem-Solving Strategies," Proceedings of the Annual Symposium on Computer Application in Medical Care, (1994), 64-68.

Vikram, K. \& Karjodkar, F. R. (2009). "Decision Support Systems in Dental Decision Making: An Introduction," Journal of Evidence-Based Dental Practice, 9 (2):73-6.

Wainwright, S. F., Shepard, K. F., Harman, L. B. \& Stephens, J. (2011). "Factors that Influencing the Clinical Decision Making of Novice and Experienced Physical Therapist," Physical Therapy, 91 (1):77-101.

Zitzman, N. U. \& Marinello, C. P. (2000). 'Treatment Plan for Restoring the Edentulous Maxilla with Implant Supported Restorations: Removable Overdenture versus Fixed Partial Design,' Journal of prosthetic Dentistry 2000; 82 (1):188-196. 